\title{
Single-dose intra-arterial neoadjuvant chemotherapy while waiting for radical hysterectomy for stage IB-IIB cervical cancer
}

\author{
KIYOSHI YOSHINO ${ }^{1}$, AYAKO HOSOI ${ }^{1}$, KEIGO OSUGA ${ }^{2}$, TAKAYUKI ENOMOTO ${ }^{1,3}$, YUTAKA UEDA ${ }^{1}$, \\ KENJIRO SAWADA $^{1}$, SEIJI MABUCHI ${ }^{1}$, EIJI KOBAYASHI ${ }^{1}$, KOJI MATSUO $^{4,5}$ and TADASHI KIMURA ${ }^{1}$ \\ Departments of ${ }^{1}$ Obstetrics and Gynecology and ${ }^{2}$ Diagnostic and Interventional Radiology, \\ Osaka University Graduate School of Medicine, Suita, Osaka 565-0871; ${ }^{3}$ Department of Obstetrics and Gynecology, \\ Niigata University Medical School, Niigata, Nigata 951-8510, Japan; ${ }^{4}$ Division of Gynecologic Oncology, \\ Department of Obstetrics and Gynecology; ${ }^{5}$ Norris Comprehensive Cancer Center, \\ University of Southern California, Los Angeles, CA 90089, USA
}

Received December 4, 2015; Accepted March 3, 2016

DOI: $10.3892 / \mathrm{mco} .2016 .846$

\begin{abstract}
Extended wait time prior to initial surgery may increase patients' anxiety. Therefore, patients may opt to receive other available treatments to inhibit tumor growth until surgery. This retrospective study describes our experience with single-dose intra-arterial neoadjuvant chemotherapy (IANAC) to more effectively utilize the wait time prior to radical hysterectomy. A total of 12 patients with International Federation of Gynecology and Obstetrics stage IB1-IIB cervical cancer were treated with single-dose IANAC prior to radical hysterectomy. Cisplatin and paclitaxel were administered intra-arterially or intravenously, respectively. The surgical outcome, prognosis and factors affecting disease recurrence were compared between these 12 patients and 57 patients in a primary surgery alone (PS) control group. As regards surgical outcome, there were no significant differences between the two groups. During the postoperative follow-up period (median, 41 months), disease recurrence was observed in 5/12 (41.6\%) IANAC cases and in 22/57 (38.5\%) PS cases (median follow-up, 54 months). There was no significant difference in disease-free survival (DFS) or 3-year survival rates between IANAC and PS (91.6 vs. 71.9\%, respectively).
\end{abstract}

Correspondence to: Dr Kiyoshi Yoshino, Department of Obstetrics and Gynecology, Osaka University Graduate School of Medicine, 2-2 Yamadaoka, Suita, Osaka 565-0871, Japan

E-mail: yoshino@gyne.med.osaka-u.ac.jp

Abbreviations: CCRT, concurrent chemoradiotherapy; CR, complete response; DFS, disease-free survival; IANAC, intra-arterial neoadjuvant chemotherapy; LD, longest diameter; MRI, magnetic resonance imaging; NAC, neoadjuvant chemotherapy; OS, overall survival; PAN, para-aortic lymph nodes; PD, progressive disease; PR, partial response; PS, primary surgery alone; RECIST, Response Evaluation Criteria in Solid Tumors; SD, stable disease; TP, paclitaxel plus cisplatin

Key words: cervical cancer, intra-arterial neoadjuvant chemotherapy, radical hysterectomy, wait time
The multivariate analysis demonstrated that wait time duration ( $\geq 45$ vs. $<45$ days) and the use of IANAC did not affect DFS. Only tumor histology (squamous vs. non-squamous) was found to be an independent prognostic factor for DFS (hazard ratio $=0.35,95 \%$ confidence inerval: $0.145-0.8967, \mathrm{P}=0.0292$ ). In addition, distal recurrence was statistically more frequent in the IANAC group compared with that in the PS group $(\mathrm{P}=0.0405)$. Therefore, single-cycle IANAC should not be performed without careful consideration.

\section{Introduction}

Uterine cervical cancer is among the most lethal malignancies affecting women worldwide. For early invasive cervical cancer, radical hysterectomy plays a pivotal role in patient outcome, and it is widely accepted that it should be expedited. However, patients must occasionally suffer long wait times due to patient overload of skilled oncologists and qualified facilities. For patients with life-threatening cancers, these progressively longer wait times prior to initial surgery represent a serious problem. The effect of delays between the onset of symptoms and the initial treatment is highly controversial and, unfortunately, cannot be investigated in randomized controlled trials due to major ethical issues.

In breast cancer, delays of 3-6 months prior to initial surgical treatment are associated with poorer survival (1). Recently, Elit et al (2) retrospectively analyzed 9,417 patients with uterine cancer regarding the association between their wait time for surgery and their prognosis, and found that patients with wait times of $>12$ weeks had worse survival compared with those with wait times of $<12$ weeks. Thus, the evidence that delays should be kept to a minimum is compelling.

Generally, neoadjuvant chemotherapy (NAC) aims to reduce the size or extent of the cancer prior to a more radical treatment, making later procedures easier and more likely to succeed. In addition, NAC may attack micrometastatic disease. NAC regimens for cervical cancer treatment have been developed, although to date there has been little clear evidence of any survival benefits for any of them. NAC 
has been performed to reduce cervical tumor bulk prior to radical hysterectomy (3). The drugs used for NAC are generally administered systemically in multiple cycles. However, Japanese researchers have been developing a special method for intra-arterial neoadjuvant chemotherapy (IANAC) delivered directly to the tumor, with the aim to expose the tumor to higher drug concentrations than would systemic intravenous delivery, while decreasing the systemic side effects (3). Thus, the idea that IANAC administered during the wait time prior to a delayed surgery may inhibit tumor growth and remove micrometastatic disease with minimum side effects, may be highly appealing to gynecological oncologists. Indeed, when informed of such potential benefits, patients often prefer an early start to this therapy, when faced with long wait times to initial surgery.

In this study, we retrospectively analyzed 12 cases of operable stage IB1-IIB cervical cancer who were treated with single-dose IANAC during their wait for subsequent radical hysterectomy, in order to better understand the efficacy and safety of IANAC for this purpose.

\section{Patients and methods}

Patients. We retrospectively reviewed the medical records of patients with uterine cervical cancer who received single-dose IANAC prior to radical hysterectomy at the Osaka University Hospital (Osaka, Japan). From January, 2002 to December, 2012, 12 patients with International Federation of Gynecology and Obstetrics (FIGO) stage IB1-IIB cervical cancer were treated with single-dose IANAC with cisplatin, followed by intravenous paclitaxel, prior to radical hysterectomy. Written informed consent for the treatment was obtained from all the patients. Due to backups, the patients were expected to wait for $>2$ months until their surgery, and each expressed a desire for an earlier start of some form of therapy. A total of 57 patients who were surgically treated without prior NAC were used as a control group. These patients comprised the primary surgery alone (PS) group, and were matched to the IANAC group for age, histology and tumor stage. The clinicopathological characteristics of the 69 patients are summarized in Table I. Age, tumor size, histology and FIGO stage were intentionally similar between the IANAC and PS groups.

Intra-arterial NAC. For the IANAC group, a paclitaxel plus cisplatin regimen (TP regimen) was applied at 4 weeks prior to subsequent radical hysterectomy. Prior to IANAC, angiography was performed to detect the tumor feeding vessels by the Seldinger technique. A microcatheter was selectively inserted into each of the uterine or hypogastric arteries. Via the microcatheter, cisplatin was injected over $2 \mathrm{~h}$, to a final dosage of $75 \mathrm{mg} / \mathrm{m}^{2}$. Intravenous hydration with normal saline $/ 5 \%$ dextrose was initiated $3 \mathrm{~h}$ prior to IANAC and was continued afterwards to maintain urine volume. After IANAC, but on the same day, paclitaxel $\left(175 \mathrm{mg} / \mathrm{m}^{2}\right)$ was administered intravenously for $3 \mathrm{~h}$. Prior to surgery, the clinical response of the tumor to IANAC was evaluated by magnetic resonance imaging (MRI) in 11 of the 12 patients; the remaining case was not evaluated due to the patient's refusal. A Piver type III radical hysterectomy was performed in all the patients in both groups.
Table I. Patient characteristics.

\begin{tabular}{lccc}
\hline Characteristics & $\begin{array}{c}\text { IANAC } \\
(\mathrm{n}=12)\end{array}$ & $\begin{array}{c}\text { PS } \\
(\mathrm{n}=57)\end{array}$ & P-value \\
\hline Age \pm SD, years & $53 \pm 7$ & $49 \pm 10$ & $0.21^{\mathrm{a}}$ \\
Tumor size \pm SD, mm & $46 \pm 6$ & $44 \pm 11$ & $0.60^{\mathrm{a}}$ \\
Histology, n (\%) & & & \\
SCC & $8(66.6)$ & $39(68.4)$ & $0.90^{\mathrm{b}}$ \\
Non-SCC & $4(33.3)$ & $18(31.5)$ & \\
FIGO stage, n (\%) & & & \\
IB1 & $1(8.3)$ & $4(7.0)$ & $0.87^{\mathrm{b}}$ \\
IB2 & $1(8.3)$ & $4(7.0)$ & 0.87 \\
IIA & $1(8.3)$ & $4(7.0)$ & 0.87 \\
IIB & $9(75.0)$ & $45(78.9)$ & 0.76 \\
\hline
\end{tabular}

at-test. 'Pearson's $\chi^{2}$-test. IANAC, intra-arterial neoadjuvant chemotherapy; PS, primary surgery alone; SD, standard deviation; SCC, squamous cell carcinoma. FIGO, International Federation of Gynecology and Obstetrics.

Operative outcome and prognosis. The operative results and prognosis were compared between the two groups. Tumor size, measured by MRI at the initial diagnostic procedure and immediately prior to surgery, was used to evaluate tumor response to NAC, and the response was classified according to the Response Evaluation Criteria in Solid Tumors, version 1.1 (4). Complete response (CR) was defined as disappearance of the tumor; partial response (PR) as a $\geq 30 \%$ decrease of the longest diameter (LD) of the tumor; progressive disease (PD) as $a \geq 20 \%$ increase of the LD; and stable disease (SD) as a change of less than the PR or PD limits.

Statistical analysis. Statistical analyses were performed using MedCalc for Windows, version 11.3.3.0 (MedCalc Software, Mariakerke, Belgium). Disease-free survival (DFS) was defined as the time (in months) from the date of completion of initial therapy to the date of a radiologically confirmed recurrence. Overall survival (OS) was calculated from the date of completion of initial therapy to the date of death. A multivariate Cox proportional hazards analysis with selected variables was used to determine the significantly important factors for recurrence. The Kaplan-Meier statistical method was used for the calculation of DFS and OS. Statistical significance was analyzed by the log-rank test. We considered the results to be statistically significant when the P-value was $<0.05$.

\section{Results}

Comparison of surgical outcomes between IANAC and PS. The results of the comparison of surgical outcomes between the IANAC and PS groups are presented in Table II. The mean wait times \pm standard deviation from diagnosis to surgery were $57 \pm 11$ and $40 \pm 15$ days for the IANAC and PS groups, respectively, indicating significantly longer wait times for the IANAC group. There were no significant differences in terms of operative time, blood loss, number of lymph nodes removed, 
Table II. Comparison of surgical outcome between IANAC and PS cervical cancer groups.

\begin{tabular}{|c|c|c|c|}
\hline Variables & IANAC (n=12) & PS $(n=57)$ & P-value \\
\hline Time to surgery $\pm \mathrm{SD}$, days & $57 \pm 11$ & $40 \pm 15$ & $0.001^{\mathrm{a}}$ \\
\hline Operative time $\pm \mathrm{SD}, \min$ & $433 \pm 132$ & $391 \pm 96$ & $0.20^{\mathrm{a}}$ \\
\hline Blood loss $\pm \mathrm{SD}, \mathrm{ml}$ & $1362 \pm 666$ & $1041 \pm 786$ & $0.19^{\mathrm{a}}$ \\
\hline Lymph nodes removed $\pm \mathrm{SD}, \mathrm{n}$ & $27 \pm 12$ & $25 \pm 12$ & $0.71^{\mathrm{a}}$ \\
\hline Transfusion, n (\%) & $8(66.6)$ & $39(68.4)$ & $0.90^{\mathrm{b}}$ \\
\hline \multicolumn{4}{|l|}{ Early complications, n (\%) } \\
\hline Surgical site infection & $3(25.0)$ & $10(17.5)$ & $0.54^{\mathrm{b}}$ \\
\hline Urinary disorder & $1(8.3)$ & $15(26.3)$ & 0.17 \\
\hline Ileus & $0(0.0)$ & $7(12.2)$ & 0.20 \\
\hline Urinary tract injury & $0(0.0)$ & $2(3.5)$ & 0.51 \\
\hline \multicolumn{4}{|l|}{ Adjuvant therapy } \\
\hline CCRT & $9(75.0)$ & $43(75.4)$ & $0.97^{\mathrm{b}}$ \\
\hline RT & $1(8.3)$ & $9(15.7)$ & 0.50 \\
\hline Chemotherapy & $2(16.6)$ & $3(5.2)$ & 0.16 \\
\hline Median follow-up, months & 41 & 54 & \\
\hline
\end{tabular}

at-test. 'Pearson's $\chi^{2}$-test. IANAC, intra-arterial neoadjuvant chemotherapy; PS, primary surgery alone; RH, radical hysterectomy; CCRT, concurrent chemoradiotherapy; RT, radiation therapy; SD, standard deviation.

or transfusion rate. As regards early complications, there was a tendency for surgical site infection to occur more frequently in IANAC (25.0\%) than in PS (17.5\%), whereas urinary disorders tended to occur less frequently in IANAC (8.3\%) than in PS (26.3\%). Ileus and urinary tract injury were not observed in IANAC; by contrast, in the PS group, ileus and urinary tract injury occurred in 12.2 and $3.5 \%$ of the patients, respectively.

Adjuvant therapy was performed in patients with deep stromal invasion, lymphovascular invasion, or positive lymph nodes. Concurrent chemotherapy and radiotherapy was applied in 9/12 (75\%) IANAC cases and in 43/57 (75\%) cases in the PS group.

In the IANAC group, 11/12 patients were evaluated with MRI immediately prior to surgery for the tumor response to IANAC. The overall clinical response rate was 54.5\% (6/11), which included a CR in 1 case $(9.0 \%)$ and a PR in 5 cases $(45.5 \%)$. The remaining 5 patients $(45.5 \%)$ had SD; no patients with PD were observed. IANAC-related toxicity was well tolerated; only 1 patient developed grade 3 neutropenia.

During the postoperative follow-up (median, 41 months) of IANAC, disease recurrence was observed in 5/12 cases $(41.6 \%)$. In the PS group, the rate was $22 / 57$ cases $(38.5 \%)$ (median, 54 months). There was no significant difference in DFS between the IANAC and PS groups (Fig. 1). In addition, there was a difference in the 3-year survival rate between the IANAC and PS groups (91.6 and 71.9\%, respectively); however, this difference was not statistically significant $(\mathrm{P}=0.1399)$.

Comparison of recurrence sites between the IANAC and PS groups. As shown in Table III, local recurrence was observed in $1 / 12$ cases $(8.3 \%$ ) in the IANAC group and in $7 / 57$ cases $(12.2 \%)$ in the PS group, with no significant difference. Among these local recurrences, 1 IANAC and 2 PS cases

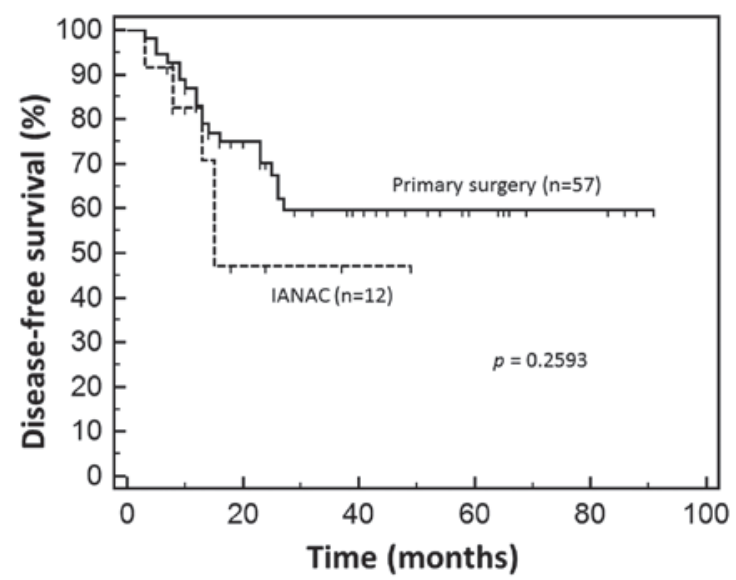

Figure 1. A Kaplan-Meier curve shows no significant difference in disease-free survival between the IANAC and control group. IANAC, intra-arterial neoadjuvant chemotherapy.

were rescued by surgery or interstitial radiotherapy, with no further evidence of tumor to date. Recurrences in the pelvic lymph nodes were observed only in the PS group (3 cases, $5.2 \%$ ). However, extrapelvic recurrence was observed more frequently in IANAC than in PS, with a statistically significant difference ( $\mathrm{P}=0.0405$; Fig. 2). Recurrences in the para-aortic lymph nodes (PAN) were observed in 2 IANAC cases $(16.6 \%)$, which was significantly higher compared with the PS group (1.7\%; $\mathrm{P}=0.021)$. Both IANAC cases with PAN recurrence were rescued by radiotherapy. Distal recurrence (except PAN) was observed in 2 IANAC (16.6\%) and 11 PS (19.3\%) cases.

Multivariate Cox proportional hazards analysis for DFS. The results of the multivariate analysis for DFS are shown in Table IV. 
Table III. Comparison of recurrence sites between the IANAC and PS groups.

\begin{tabular}{lccc}
\hline Sites of recurrence, $\mathrm{n}(\%)$ & IANAC $(\mathrm{n}=12)$ & PS $(\mathrm{n}=57)$ & P-value \\
\hline Local recurrence & $1(8.3)$ & $7(12.2)$ & $0.67^{\mathrm{a}}$ \\
Pelvic lymph nodes & $0(0.0)$ & $3(5.2)$ & $0.41^{\mathrm{a}}$ \\
PAN & $2(16.6)$ & $1(1.7)$ & $0.02^{\mathrm{a}}$ \\
Distal recurrence (except PAN) & $2(16.6)$ & $11(19.3)$ & $0.83^{\mathrm{a}}$ \\
Total & $5(41.6)$ & $22(38.5)$ & $0.54^{\mathrm{a}}$ \\
\hline
\end{tabular}

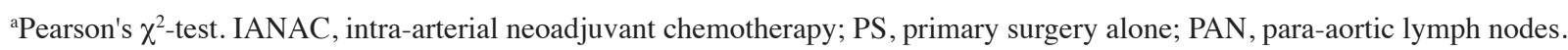

Table IV. Multivariate Cox proportional hazards analysis for DFS in cervical cancer.

\begin{tabular}{|c|c|c|c|}
\hline Variables & HR & $95 \% \mathrm{CI}$ of $\mathrm{HR}$ & P-value \\
\hline Age, years & & & 0.76 \\
\hline$\geq 50$ & 0.86 & $0.3471-2.1719$ & \\
\hline$<50$ & 1 & & \\
\hline Wait time, days & & & 0.88 \\
\hline$\geq 45$ & 0.93 & $0.3567-2.4326$ & \\
\hline$<45$ & 1 & & \\
\hline Tumor size, cm & & & 0.58 \\
\hline$\geq 5$ & 0.79 & $0.3508-1.8003$ & \\
\hline$<5$ & 1 & & \\
\hline IANAC & & & 0.4 \\
\hline Yes & 1.61 & $0.5317-4.9045$ & \\
\hline No & 1 & & \\
\hline Histology & & & 0.0292 \\
\hline SCC & 0.35 & $0.1415-0.8967$ & \\
\hline Non-SCC & 1 & & \\
\hline Lymph node metastasis & & & 0.15 \\
\hline Positive & 2.01 & $0.7719-5.2549$ & \\
\hline Negative & 1 & & \\
\hline
\end{tabular}

DFS, disease-free survival; IANAC, intra-arterial neoadjuvant chemotherapy; SCC, squamous cell carcinoma; HR, hazard ratio; CI, confidence interval.

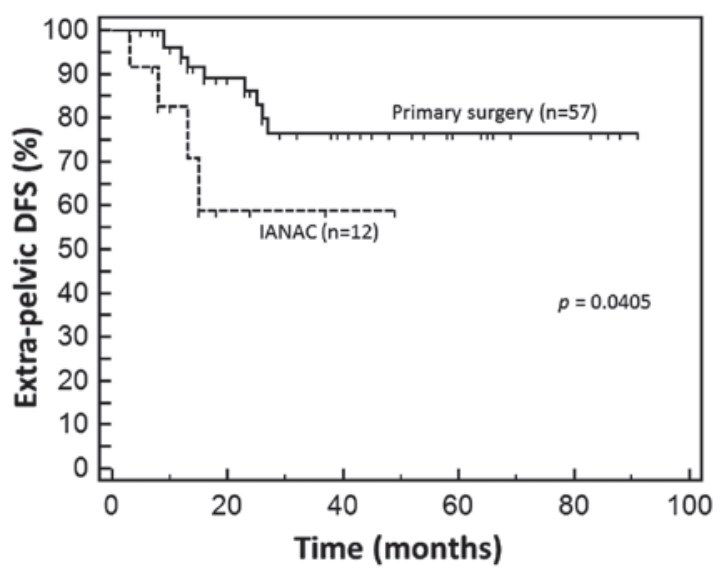

Figure 2. A Kaplan-Meier curve shows the disease-free survival (DFS) in terms of extrapelvic disease. Distal recurrence was observed more frequently in the intra-arterial neoadjuvant chemotherapy (IANAC) group than in the primary surgery alone group, with a statistically significant difference $(\mathrm{P}=0.0405)$.
The type of tumor histology (squamous vs. non-squamous) was determined to be an independent prognostic factor for DFS (hazard ratio $=0.35,95 \%$ confidence interval: $0.1415-0.8967$, $\mathrm{P}=0.0292)$. However, age ( $\geq 50$ vs. $<50$ years), wait time until surgery ( $\geq 45$ vs. $<45$ days), initial tumor size ( $\geq 5$ vs. $<5 \mathrm{~cm}$ ), and lymph node metastasis status (positive vs. negative) did not affect DFS in our series.

\section{Discussion}

Our study demonstrated that single-dose IANAC, delivered while waiting for radical hysterectomy, was not associated with an objective benefit for patients with stage IB-IIB cervical cancer. In our study, there were no significant differences in DFS or 3-year survival rates between the IANAC and PS groups. A multivariate analysis revealed that the wait time until surgery ( $\geq 45$ vs. $<45$ days) or the use or of IANAC did 
not affect DFS. Only the type of histology (squamous vs. non-squamous) was found to be an independent prognostic factor for DFS. Surprisingly, we observed distal recurrences more frequently in the IANAC rather than in the PS control group, with a statistically significant difference. This may indicate that IANAC may promote new spreading of tumor cells, rather than the hoped for effect of eradicating pre-existing micrometastases.

Previous studies, usually performed with 3 cycles of platinum and taxanes, have shown the benefits of IANAC for controlling bulky cervical cancer. IANAC was effective at reducing tumor bulk, with reduced toxicity (relative to intravenous treatments), with safety and improved prognosis (3,5-9).

In ovarian cancer, $>3$ cycles of NAC are required to efficiently control the primary as well as the distant tumors. In early-stage ovarian cancer, 6 cycles of adjuvant carboplatin and paclitaxel significantly reduced recurrence, compared with 3 cycles ). This result suggested that, to vanquish micrometastases of hardier cancer cells, repeated administrations of chemotherapy are required. Therefore, if IANAC use is inevitable, multiple cycles of administration is clearly preferable to a single dose, as was attempted in this study.

Due to the high ratio of patients with technically difficult surgical needs to the low numbers of fully skilled gynecologic oncologists and hospitals with advanced facilities, wait times for cancer surgeries have increased, causing serious problems for the patients. These longer wait times for surgery may cause the patient anxiety, thereby negatively affecting prognosis. For patients to cope with longer wait times, concurrent chemoradiotherapy (CCRT) may be the most reasonable option. The evidence for the efficacy of CCRT in cervical cancer is well established; therefore, in order to start therapy early, some patients have been treated with CCRT instead of surgery. However, some patients prefer to undergo surgery instead of CCRT, due to the long-lasting side effects of radiation. In such cases, multiple cycles of IANAC, followed by radical hysterectomy, may be a viable option. The limitations of our study were the relatively small number of IANAC cases, the retrospective design that may cause bias, and the relatively short follow-up period.

In conclusion, although single-dose IANAC may help reduce patient anxiety and permit earlier surgery (compared with multiple cycles of IANAC), its survival benefit is equivocal and it should not be performed lightly. Furthermore, it is recommended that single-cycle IANAC should not be performed without careful consideration. It is likely that multiple-cycle IANAC will perform better than a single dose for controlling distant micrometastases, as higher response rates and better prognosis were previously reported using $\geq 3$ cycles.

\section{Acknowledgements}

We would like to thank Dr G.S. Buzard for his helpful comments and editing.

\section{References}

1. Richards MA, Westcombe AM, Love SB, Littlejohns P and Ramirez AJ: Influence of delay on survival in patients with breast cancer: A systematic review. Lancet 353: 1119-1126, 1999.

2. Elit LM, O'Leary EM, Pond GR and Seow HY: Impact of wait times on survival for women with uterine cancer. J Clin Oncol 32: 27-33, 2014

3. Sugiyama T, Nishida T, Hasuo Y, Fujiyoshi K and Yakushiji M: Neoadjuvant intraarterial chemotherapy followed by radical hysterectomy and/or radiotherapy for locally advanced cervical cancer. Gynecol Oncol 69: 130-136, 1998.

4. Eisenhauer EA, Therasse P, Bogaerts J, Schwartz LH, Sargent D, Ford R, Dancey J, Arbuck S, Gwyther S, Mooney M, Rubinstein L, Shankar L, Dodd L, Kaplan R, Lacombe D and Verweij J: New response evaluation criteria in solid tumours: revised RECIST guideline (version 1.1). Eur J Cancer 45: 228-247, 2009.

5. Yamakawa Y, Fujimura M, Hidaka T, Hori S and Saito S: Neoadjuvant intraarterial infusion chemotherapy in patients with stage IB2-IIIB cervical cancer. Gynecol Oncol 77: 264-270, 2000.

6. Aoki Y, Sato T, Watanabe M, Sasaki M, Tsuneki I and Tanaka K: Neoadjuvant chemotherapy using low-dose consecutive intraarterial infusions of cisplatin combined with 5-fluorouracil for locally advanced cervical adenocarcinoma. Gynecol Oncol 81: 496-499, 2001

7. Motoyama S, Hamana S, Ku Y, Laoag-Fernandez JB, Deguchi M, Yoshida S, Tominaga M, Iwasaki T, Ohara $\mathrm{N}$ and Maruo T: Neoadjuvant high-dose intraarterial infusion chemotherapy under percutaneous pelvic perfusion with extracorporeal chemofiltration in patients with stages IIIa-IVa cervical cancer. Gynecol Oncol 95: 576-582, 2004.

8. Terai Y, Kanemura M, Sasaki H, Tsunetoh S, Tanaka Y, Yamashita Y, Yamamoto K, Narabayashi I and Ohmichi M: Long-term follow-up of neoadjuvant intraarterial chemotherapy using an original four-lumen double-balloon (4L-DB) catheter for locally advanced uterine cervical cancer. Int J Clin Oncol 14: 56-62, 2009.

9. Kaku S, Takahashi K, Murakami Y, Wakinoue S, Nakagawa T, Shimizu Y, Kita N, Noda Y and Murakami T: Neoadjuvant intraarterial chemotherapy for stage IIB-IIIB cervical cancer in Japanese women. Exp Ther Med 1: 651-655, 2010.

10. Bell J, Brady MF, Young RC, Lage J, Walker JL, Look KY, Rose GS and Spirtos NM; Gynecologic Oncology Group: Randomized phase III trial of three versus six cycles of adjuvant carboplatin and paclitaxel in early stage epithelial ovarian carcinoma: A Gynecologic Oncology Group study. Gynecol Oncol 102: 432-439, 2006. 